\title{
MOLÉCULAS PROTROMBÓTICAS EN NIÑOS CON DIABETES MELLITUS TIPO 1
}

\section{STUDY OF PROTHROMBOTIC MOLECULES IN TYPE 1 DIABETES MELLITUS CHILDREN}

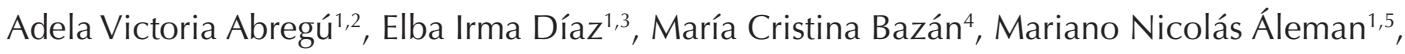 \\ María Constanza Luciardi ${ }^{1,6}$, Ana Carolina Mariani ${ }^{1,7}$
}

\section{RESUMEN}

Introducción: la hiperglucemia contribuye a cambios moleculares que alteran la hemostasia.

Objetivos: determinar moléculas circulantes que indiquen la presencia de un estado protrombótico en una población infanto juvenil con diabetes mellitus tipo 1 (DM1), sin manifestación clínica de enfermedad vascular, y compararla con una población control. Pacientes y métodos: se estudiaron 35 pacientes con DM1, de $11,0 \pm 2,5$ años de edad y 3,7 $\pm 2,0$ años de evolución de la enfermedad, sin complicaciones vasculares y 20 controles sanos de edad, sexo e IMC semejantes. Se determinaron: fibrinógeno (Fg), inhibidor del activador del plasminógeno 1 (PAI-1), antígeno del factor von Willebrand (FvW:Ag), ligando CD40 soluble (sCD40L) y pruebas globales de coagulación como recuento de plaquetas, tiempo de protrombina (TP) y tiempo de tromboplastina parcial activado (APTT). El control glucémico se evaluó mediante glucemia en ayunas y A1c, y se descartó la presencia de retinopatía y nefropatía. Los datos se analizaron con el programa SPSS 20 para Windows y se expresaron como media $\pm D E$. El coeficiente de Pearson se usó para investigar las correlaciones entre las variables estudiadas.

Resultados: los pacientes con DM1 presentaron valores significativamente mayores de $\mathrm{Fg}(308 \pm 66$ vs $246 \pm 18 \mathrm{mg} / \mathrm{dL}, \mathrm{p}=0,0001)$, PAl- $1(41,6 \pm 12$ vs $11,7 \pm 1,0 \mathrm{ng} / \mathrm{mL}, \mathrm{p}=0,0001)$, FvW:Ag $(284 \pm 55$ vs $121 \pm 19 \%, p=0,0001)$ y sCD40L $(1608 \pm 109$ vs $149 \pm 17 \mathrm{pg} / \mathrm{mL}$, $p=0,0001)$. Sin embargo las pruebas globales de hemostasia no mostraron diferencias entre ambos grupos. El PAI-1 y SCD40L se correlacionaron con glucemia, A1c, Fg y FvW:Ag.

Conclusiones: los niveles elevados de Fg, PAl-1, FvW:Ag y sCD40L sugieren la presencia de un estado protrombótico en la población infanto juvenil con DM1.

Palabras clave: diabetes; estado protrombótico; PAI-1; factor von Willebrand; sCD40L.

Revista de la Sociedad Argentina de Diabetes 2019; Vol. 53 (70-75)

\section{ABSTRACT}

Introduction: hyperglycemia contributes to molecular changes that alter hemostasis.

Objectives: to determine molecules of a prothrombotic state in a child-juvenile population with type 1 diabetes (T1D), without clinical manifestation of vascular disease, and compare it with a control population.

Patients and methods: thirty-five patients with T1D (11.0 \pm 2.5 years and $3.7 \pm 2.0$ years of disease duration), without vascular complications and 20 healthy controls were studied. Plasma fibrinogen (Pf), plasminogen activator inhibitor 1 (PAl-1), von Willebrand factor antigen VWF:Ag and soluble CD40 ligand (sCD4OL) and coagulation global tests such as platelet count, prothrombin time (PT), activated partial thromboplastin time (APTT) were determined. The data obtained were analized by Statistics SPSS 20 software and were expressed as the mean \pm standard desviation. Pearson coefficient was used to investigate correlations between variables.

Results: diabetic patients presented significantly higher values of glycaemia, A1c, Fg (308 \pm 66 vs 246 $\pm 18 \mathrm{mg} / \mathrm{dL}, p=0.0001)$, PAl-1 (41.6 \pm 12 vs $11.7 \pm 1,0 \mathrm{ng} / \mathrm{mL}, p=0.0001), v W F: A g(284 \pm 55$ vs $121 \pm 19 \%, p=0.0001)$ and SCD $40 \mathrm{~L}(1608 \pm 109 \mathrm{vs} 149 \pm 17 \mathrm{pg} / \mathrm{mL}$, $p=0.0001$ ). However, overall hemostasis tests showed no differences between both groups, PAl-1 and SCD4OL correlated with glycemia, A1c, Fg and vWF:Ag.

Conclusions: high levels of Fg, PAl-1, VWF:Ag and SCD4OL suggest the presence of a prothrombotic state in the infant population juvenil with DT1.

Key words: diabetes; prothrombotic state; PAl-1; von Willebrand factor; sCD4OL.

Revista de la Sociedad Argentina de Diabetes 2019; Vol. 53 (70-75)
1 Cátedra de Práctica Profesional, Facultad de Bioquímica, Química y Farmacia, Universidad Nacional de Tucumán, Tucumán, Argentina

2 Dra. en Bioquímica, Prof. Titular Práctica Profesional, Tucumán, Argentina

3 Bioquímica, Profesora Asociada Práctica Profesional, Tucumán, Argentina

4 Dra. en Medicina, Jefa del Servicio de Endocrinología, Hospital del Niño Jesús (SIPROSA), Tucumán, Argentina

5 Dr. en Bioquímica, Docente Práctica Profesional, Tucumán, Argentina
6 Dra. en Bioquímica, Docente Práctica Profesional, Tucumán, Argentina

7 Bioquímica especializada en Hematología, Docente Práctica Profesional, Tucumán, Argentina

Contacto de la autora: Adela Victoria Abregú E-mail: avabregu@gmail.com

Correspondencia: Pje. Puerto Argentino 1368 (CP4000)

San Miguel de Tucumán, Tucumán, Argentina

Fecha de trabajo recibido: 17/02/19

Fecha de trabajo aceptado: 07/05/19

Conflictos de interés: los autores declaran que no existe conflicto de interés 


\section{INTRODUCCIÓN}

La diabetes mellitus tipo 1 (DM1) es una de las condiciones endocrinas y metabólicas más comunes en la infancia y su incidencia está aumentando y puede duplicar la carga de la enfermedad en 2020, principalmente en los jóvenes y aquellos con susceptibilidad genética moderada!

Se ha demostrado que la hiperglucemia puede contribuir a cambios moleculares que alteran la hemostasia e inducen a un microambiente proinflamatorio, protrombótico y antifibrinolítico ${ }^{2}$. Los principales componentes del sistema hemostático cuya concentración o actividad se alteran por la hiperglucemia incluyen el fibrinógeno (Fg), el inhibidor-1 del activador del plasminógeno (PAl-1, sus siglas en inglés), el factor von Willebrand (FvW) y la antitrombina.

Los niveles elevados de Fg se han asociado con la enfermedad arterial coronaria en pacientes con DM1. La hiperfibrinogenemia conduce a una mayor actividad coagulante y a un aumento en la viscosidad sanguínea. También el Fg contribuye a la formación de la placa ateromatosa y a su activación. Adicionalmente la hiperfibrinogenemia es un indicador de cambios vasculares inflamatorios y de disfunción endotelial ${ }^{3}$.

EI PAI-1 -producido por las plaquetas, células endoteliales y tejido adiposo- es el inhibidor endógeno más importante del sistema fibrinolítico. Se ha observado una clara asociación entre niveles plasmáticos elevados de PAI-1 y enfermedades protrombóticas como hipertensión, obesidad, resistencia a la insulina y diabetes ${ }^{4,5}$.

El FvW es una glicoproteína producida selectivamente por células endoteliales y megacariocitos. Cumple un papel fundamental en la hemostasia dado que protege el factor VIII de la degradación proteolítica y también en la trombosis porque favorece la adhesión y agregación plaquetaria al endotelio dañado. Además el FvW es un mediador de la inflamación vascular porque induce al reclutamiento y extravasación de leucocitos, y activa la cascada del complemento. Recientemente se ha propuesto como un marcador pronóstico de enfermedades metabólicas y cardiovasculares como diabetes, infarto de miocardio o accidente cerebrovascular ${ }^{6,7}$.

En la diabetes el sistema fibrinolítico es relativamente lento debido a que las redes de fibrina que forman el trombo son más resistentes a la degradación y por el aumento de los niveles de PAI-18,9.

Por otra parte, el ligando CD40L y su forma soluble (sCD40L), pertenecientes a la superfamilia del factor de necrosis tumoral, son moléculas con propiedades proinflamatorias y protrombóticas que se unen a su receptor CD40. Se expresan en una variedad de células como endoteliales, macrófagos, linfocitos y plaquetas. El sistema CD40/ CD40L tiene un papel relevante en la inflamación subclínica, en la patogénesis de la aterosclerosis y de la trombosis. Diversos estudios demostraron que niveles elevados del CD40L se asocian a factores de riesgo cardiovascular como diabetes, hipercolesterolemia, hipertensión y obesidad ${ }^{10,11}$.

Aunque las complicaciones de la DM1 en la infancia son poco frecuentes, anormalidades funcionales y estructurales del endotelio como niveles elevados de moléculas de adhesión o el incremento del espesor de la íntima-media de la arteria carótida, están presentes en estos pacientes antes que las manifestaciones clínicas de vasculopatía ${ }^{12,13,14}$. Sin embargo las investigaciones de moléculas protrombóticas en este grupo etario aún son insuficientes.

El objetivo de este trabajo fue investigar la presencia de un estado protrombótico en una población infanto juvenil con DM1 mediante la determinación de Fg, PAl-1, antígeno del FvW (FvW:Ag), sCD40L y pruebas globales como recuento de plaquetas (RP), tiempo de protrombina (TP) y tiempo de tromboplastina parcial activado (APTT, sus siglas en inglés). Asimismo se analizó la relación de estos parámetros con el grado de control glucémico, el tiempo de evolución de la enfermedad y la asociación con las variables estudiadas.

\section{PACIENTESY MÉTODOS \\ Población estudiada}

En este trabajo, de diseño observacional, analítico y de corte transversal, se incluyeron 35 pa-

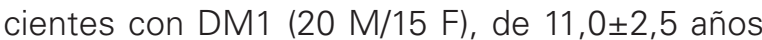
de edad, con un tiempo de evolución de la enfermedad de 3,7 $\pm 2,0$ años, sin complicaciones vasculares clínicamente demostrables, que concurrieron al Servicio de Endocrinología del Hospital del Niño Jesús de San Miguel de Tucumán, durante el período 2015-2017, y se los comparó con 20 sujetos sanos de edad, sexo e IMC semejantes y sin antecedentes familiares de diabetes. Todos los pacientes fueron sometidos a una evaluación clínica completa y consignaron datos de peso, taIla, IMC, tiempo de evolución de la enfermedad y antecedentes familiares de diabetes y/o patología cardiovascular. El peso se midió con una balanza de palanca, con ropa liviana y sin calzado. La talla se determinó en un altímetro, con los talones 
juntos, los hombros relajados y ambos brazos a los costados del cuerpo. El IMC se calculó con la fórmula Quetelet (peso/talla²). Los niños con DM1 fueron tratados con insulina glargina (Lantus $\left.{ }^{\circledR}\right)$ a

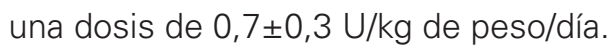

La ausencia de retinopatía se confirmó por examen oftalmológico con estudio de fondo de ojo. La presencia de nefropatía se descartó mediante la determinación de albumina urinaria por método inmunoturbidimétrico (DCA 2000, Siemens, EE.UU.). Los niños incluidos en este protocolo presentaron niveles normales de presión arterial para edad y sexo según tablas de la Sociedad Argentina de Diabetes (SAD), como así también los lípidos séricos. Asimismo se excluyeron del presente estudio aquellos con enfermedades inflamatorias o infecciosas, patologías hepáticas, renales agudas o crónicas, síndrome de malabsorción, hipotiroidismo u otra endocrinopatía.

\section{Determinaciones bioquímicas}

La extracción de sangre se realizó entre las 8 y las 10 de la mañana por punción venosa sin utilizar lazo, previo ayuno de $8 \mathrm{~h}$. El RP se realizó en sangre anticoagulada con EDTA al 10\%, en contador hematológico (Sysmex). En plasma pobre en plaquetas, obtenido con citrato de sodio 3,2\% $(0,109 \mathrm{M})$ en la proporción $1 / 9$ y centrifugado a 3.000 rpm 15 minutos, se midieron: TP, APTT y Fg dentro de las $4 \mathrm{~h}$ de extraída la muestra en un autoanalizador ACL 300 Research (Instrumentation Laboratory, Italia). Los niveles de PAI-1 y FvW:Ag se determinaron por ELISA (Asserachrom, Diagnostica Stago). En suero se midieron los niveles de sCD40L (método ELISA, PeproTech, EE.UU.). El grado de control glucémico se evaluó por valores de glucemia en ayunas (método enzimático, Wiener Lab., Argentina) y A1c (DCA 2000, Siemens, EE.UU.). También se analizaron los valores de glucemia capilar individuales chequeados con los monitores de los pacientes y resultó un promedio de $174 \mathrm{mg} / \mathrm{dL}$ antes del desayuno, $190 \mathrm{mg} /$ $\mathrm{dL}$ antes del almuerzo y $210 \mathrm{mg} / \mathrm{dL}$ antes de la cena. De acuerdo a la recomendación de la Asociación Americana de Diabetes (ADA) se consideró buen control glucémico (BCG) un valor de $\mathrm{A} 1 \mathrm{C}$ $<8 \%$ y pobre control glucémico (PCG) $\mathrm{A} 1 \mathrm{c} \geq 8 \%$.

Los datos se analizaron con el programa SPSS 20 para Windows y se expresaron como la media $\pm D E$. Para comparar ambos grupos se usó el test de hipótesis para muestras independientes y el coeficiente de
Pearson para investigar las correlaciones entre las variables. Un valor de $\mathrm{p}<0,05$ se consideró significativo.

Para la realización de este trabajo se contó con la aprobación del Comité de Docencia e Investigación del Hospital y la firma de los consentimientos informados por los padres y/o tutores de los pacientes.

\section{RESULTADOS}

En la Tabla 1 se observan las características clínicas y bioquímicas de los grupos estudiados. Los pacientes diabéticos presentaron valores significativamente mayores de glucemia, A1c, Fg, PAl1, FvW:Ag y sCD40L. Sin embargo las pruebas globales de hemostasia no mostraron diferencias significativas entre ambos grupos.

El 27\% $(n=9)$ de los niños diabéticos presentó un buen control glucémico y el $73 \%(n=26)$ restante un pobre control glucémico. Cuando se analizaron las variables según el grado de control glucémico, sólo se encontró diferencia significativa en los valores de sCD40L entre diabéticos con BCG y PCG (Tabla 2).

Cuando se investigaron las variables según el tiempo de evolución de la enfermedad ( $\leq 3$ años y $>3$ años) no se observaron diferencias significativas en ninguna de las moléculas estudiadas (Tabla 3).

En el análisis de las correlaciones se encontró que PAl-1 y sCD40L correlacionaron positivamente con glucemia, A1c, Fg y FvW (Tablas 4 y 5).

\begin{tabular}{|l|r|r|r|}
\hline & Diabéticos & Controles & p \\
\hline $\mathrm{N}$ & 35 & 20 & --- \\
\hline Varones/mujeres & $20 / 15$ & $13 / 7$ & -- \\
\hline Edad (años) & $11,0 \pm 2,5$ & $10,2 \pm 1,6$ & $\mathrm{NS}$ \\
\hline IMC (kg/m $\left.{ }^{2}\right)$ & $19,4 \pm 4,0$ & $18,7 \pm 1,5$ & $\mathrm{NS}$ \\
\hline Evolución (años) & $3,7 \pm 2,0$ & ---- & --- \\
\hline Glucemia (mg/dL) & $210 \pm 109$ & $77 \pm 8$ & 0,0001 \\
\hline A1c (\%) & $10,4 \pm 3,0$ & $5,8 \pm 0,4$ & 0.0001 \\
\hline Colesterol total (mg/dL) & $167 \pm 39$ & $156 \pm 28$ & $\mathrm{NS}$ \\
\hline HDL-colesterol (mg/dL) & $45 \pm 12$ & $42 \pm 8$ & $\mathrm{NS}$ \\
\hline LDL-colesterol (mg/dL) & $107 \pm 39$ & $99 \pm 30$ & $\mathrm{NS}$ \\
\hline Triglicéridos (mg/dL) & $90 \pm 48$ & $87 \pm 16$ & $\mathrm{NS}$ \\
\hline Tiempo protrombina (\%) & $86 \pm 15$ & $90 \pm 9$ & $\mathrm{NS}$ \\
\hline APTT (seg) & $45 \pm 6$ & $42 \pm 5$ & $\mathrm{NS}$ \\
\hline Recuento plaquetas $\left(\mathrm{x} 10^{3} / \mathrm{uL}\right)$ & $286 \pm 37,2$ & $209 \pm 50$ & $\mathrm{NS}$ \\
\hline Fibrinógeno (mg/dL) & $308 \pm 66$ & $246 \pm 18$ & 0,0001 \\
\hline PAl-1 (ng/mL) & $41,6 \pm 12,0$ & $11,7 \pm 1,0$ & 0,0001 \\
\hline FvW:Ag $\%$ ) & $284 \pm 55$ & $121 \pm 19$ & 0,0001 \\
\hline sCD40L (pg/mL) & $1.608 \pm 149$ & $109 \pm 17$ & 0,001 \\
\hline
\end{tabular}

Los resultados se expresan como media $\pm D S$. $p$ significativo $<0,05 ;$ NS: no significativo.

Tabla 1: Características clínicas y bioquímicas de los grupos estudiados. 


\begin{tabular}{|l|r|r|r|}
\hline & BCG $(\mathbf{n = 9})$ & PCG $(\mathbf{n = 2 6 )}$ & \multicolumn{1}{c|}{$\boldsymbol{p}$} \\
\hline A1c (\%) & $7,2 \pm 0,3$ & $11,5 \pm 2,7$ & 0,0001 \\
\hline Tiempo protrombina (\%) & $88 \pm 8$ & $84 \pm 18$ & 0,69 \\
\hline APTT (seg) & $45 \pm 3$ & $46 \pm 8$ & 0,77 \\
\hline Recuento plaquetas (x 103/uL) & $274 \pm 47$ & $290 \pm 36$ & 0.40 \\
\hline Fibrinógeno (mg/dL) & $341 \pm 64$ & $290 \pm 63$ & 0,13 \\
\hline PAl-1 (ng/mL) & $43,8 \pm 12,1$ & $40,4 \pm 13,3$ & 0,61 \\
\hline FvW:Ag (\%) & $272 \pm 39$ & $288 \pm 58$ & 0,58 \\
\hline sCD40L (pg/mL) & $1.758 \pm 73$ & $1.560 \pm 143$ & 0,02 \\
\hline
\end{tabular}

Los resultados se expresan como media $\pm D S$.

p significativo $<0,05 ; B C G$ : buen control glucémico ( $A 1 c \leq 8 \%)$; PCG: pobre control glucémico (A1C >8\%).

Tabla 2: Moléculas protrombóticas en diabéticos según el grado de control glucémico.

\begin{tabular}{|l|r|r|r|}
\hline & $\begin{array}{c}\leq \text { 3 años } \\
(\mathbf{n = 1 6 )}\end{array}$ & $\begin{array}{c}>\text { >3 años } \\
(\mathbf{n = 1 9 )}\end{array}$ & $\boldsymbol{p}$ \\
\hline Tiempo protrombina (\%) & $83 \pm 12$ & $88 \pm 18$ & 0,44 \\
\hline APTT (seg) & $43 \pm 4$ & $47 \pm 7$ & 0,20 \\
\hline Recuento plaquetas (x 103/uL) & $288 \pm 40$ & $283 \pm 35$ & 0.73 \\
\hline Fibrinógeno (mg/dL) & $320 \pm 73$ & $297 \pm 60$ & 0,42 \\
\hline PAI-1 (UA/mL) & $46,1 \pm 11,4$ & $38,6 \pm 11,9$ & 0,17 \\
\hline FvW:Ag (\%) & $264 \pm 50$ & $293 \pm 60$ & 0,27 \\
\hline sCD40L (pg/mL) & $1546 \pm 207$ & $1633 \pm 124$ & 0,34 \\
\hline
\end{tabular}

Los resultados se expresan como media $\pm D S$. $p$ significativo $<0,05$

Tabla 3: Moléculas protrombóticas en diabéticos según el tiempo de evolución de la enfermedad.

\begin{tabular}{|l|r|r|}
\hline \multicolumn{3}{|c|}{ PAI-1 } \\
\hline & \multicolumn{1}{c|}{ r } & \multicolumn{1}{c|}{$\boldsymbol{p}$} \\
\hline Glucemia & 0,40 & 0,02 \\
\hline A1c & 0,48 & 0,009 \\
\hline sCD40L & 0,92 & 0,0001 \\
\hline Fibrinógeno & 0,48 & 0,007 \\
\hline FvW:Ag & 0,72 & 0,0001 \\
\hline
\end{tabular}

Se aplicó el coeficiente de correlación de Pearson. $p$ significativo $<0,05$.

Tabla 4: Correlación entre PAI-1 y otras variables.

\begin{tabular}{|l|r|r|}
\hline \multicolumn{3}{|c|}{ sCD40L } \\
\hline & \multicolumn{1}{c|}{$\boldsymbol{r}$} & \multicolumn{1}{c|}{$\boldsymbol{p}$} \\
\hline Glucemia & 0,56 & 0,004 \\
\hline A1c & 0,64 & 0,001 \\
\hline PAI-1 & 0,92 & 0,0001 \\
\hline Fibrinógeno & 0,43 & 0,02 \\
\hline FvW:Ag & 0,85 & 0,0001 \\
\hline
\end{tabular}

Se aplicó el coeficiente de correlación de Pearson. p significativo $<0,05$.

Tabla 5: Correlación entre sCD40L y otras variables.

\section{DISCUSIÓN}

Las complicaciones vasculares son las principales causas de morbilidad y mortalidad en los pacientes diabéticos. Al presente la información sobre los desórdenes de la coagulación y la fibrinólisis en la DM1 infanto juvenil es limitada ${ }^{15}$.

En nuestro trabajo no hubo diferencias en el número de plaquetas, elTP y APTT en la población de niños diabéticos estudiada comparada con los controles sanos. Resultados semejantes obtuvieron otros autores ${ }^{16}$. No obstante hay evidencias de disfunción plaquetaria y APTT acortados en diabéticos adultos comparados con no diabéticos que podrían reflejar un estado de hipercoagulabilidad potencialmente asociado a un riesgo protrombótico ${ }^{17,18,19}$.

Los resultados más relevantes de este estudio muestran niveles aumentados de Fg, PAl-1, FvW:Ag y sCD40L en los niños diabéticos. En coincidencia, varios estudios realizados en adultos y niños con DM1 sugirieron la presencia de anomalías en la hemostasia. De este modo, Carmassi et al. encontraron que los pacientes DM1 presentaban niveles más altos de Fg, factor VII, complejos trombina-antitrombina y PAI1 y menor actividad del activador del plasminógeno tisular (tPA, sus siglas en inglés) en comparación con sujetos controles ${ }^{16}$. Otros investigadores informaron que, comparados con los controles sanos, los niveles plasmáticos de Fg, FvW, sICAM-1 y de PAl-1 eran notablemente mayores en jóvenes no fumadores con DM1 sin evidencia clínica de enfermedad macrovascular $^{20}$. Adly et al. confirmaron niveles elevados de PAl-1 en niños y adolescentes con DM1 respecto de los controles, y al comparar diabéticos con y sin complicaciones vasculares, el PAl-1 fue mayor en aquellos con complicaciones ${ }^{9}$. Recientemente un trabajo evaluó el perfil de la hemostasia en niños con DM1 y halló hipercoagulabilidad y aumento en la firmeza máxima del coágulo²1.

Todos estos estudios revelan desórdenes hemostáticos en pacientes con DM1. Varios mecanismos contribuyen al estado protrombótico, incluidos disfunción endotelial, hipercoagulabilidad e hipofibrinólisis. Además la hiperglucemia crónica conduce a la glicación de las proteínas involucradas en la fibrinólisis al afectar la estructura fisiológica del coágulo y hacerlo más resistente a la degradación por la plasmina ${ }^{22,23}$. También la hiperglucemia activa la vía metabólica del poliol y la proteinquinasa $\mathrm{C}$, y aumenta el estrés oxidativo. Todos estos procesos inician cambios vasculares que alteran el sistema hemostático ${ }^{24,25}$. 
En la actualidad las investigaciones del SCD40L en niños con DM1 son escasas y discordantes. En el presente trabajo los niveles séricos del SCD40L estuvieron elevados en los niños diabéticos sin complicaciones vasculares y se correlacionaron positivamente con la A1c. Estos resultados coinciden con los de otros autores que informaron niveles séricos incrementados de SCD40L en niños con DM1, particularmente en aquellos con complicaciones microvasculares, y detectaron una correlación entre este ligando y el grado de control glucémico ${ }^{26,27}$. Por el contrario, Targher et al. en un estudio realizado en jóvenes diabéticos normotensos, no fumadores, con buen control glucémico y sin complicaciones vasculares, observaron niveles séricos de SCD40L similares a los del grupo

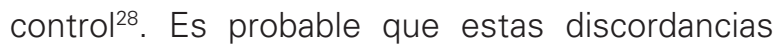
puedan explicarse por las características de las distintas poblaciones estudiadas, incluyendo presencia de complicaciones crónicas, grado de control glucémico y perfil de riesgo aterogénico de los pacientes.

Una de las limitaciones de este trabajo fue el número de niños estudiados, por lo cual los datos obtenidos no pueden generalizarse, sin embargo podrían considerarse resultados preliminares para niños diabéticos del noreste argentino (NOA). Por otro lado, el diseño de corte transversal permite establecer asociación pero no causalidad. Se necesitan futuros estudios longitudinales que incluyan un mayor número de pacientes para confirmar los resultados y evaluar la utilidad clínica de estos biomarcadores con el fin de identificar el riesgo protrombótico en los niños diabéticos.

\section{CONCLUSIONES}

En la población infanto juvenil con DM1 estudiada los resultados obtenidos muestran niveles elevados de Fg, PAI-1, FvW:Ag y sCD40L, y sugieren la presencia de un estado protrombótico. Los niños con DM1 se enfrentan a largos años de hiperglucemia con el riesgo de desarrollo temprano de complicaciones micro y macrovasculares. Por estos motivos la detección precoz de estas moléculas contribuirá a implementar estrategias para prevenir dichas alteraciones desde la infancia.

\section{BIBLIOGRAFÍA}

1. Vehik K, Dabelea D. The changing epidemiology of type 1 diabetes: why is it going through the roof? Diabetes Metab Res Rev 2011; 27(1):3-13.

2. Targher G, Chonchol M, Zoppini G, et al. Hemostatic disorders in type 1 diabetes mellitus. Semin Thromb Hemost 2011; 37(1):58-65.

3. Rodrigues TC, Snell-Bergeon JK, Maahs DM, et al. Higher fibrinogen levels predict progression of coronary artery calcification in adults with type 1 diabetes. Atherosclerosis 2010; 210(2):671-673.

4. Alessi MC, Poggi M, Juhan-Vague I. Plasminogen activator inhibitor-1, adipose tissue and insulin resistance. Curr Opin Lipidol 2007; 18(3):240-245.

5. Cesari M, Pahor M, Incalzi RA. Plasminogen activator inhibitor-1 (PAI-1): a key factor linking fibrinolysis and age-related subclinical and clinical conditions. Cardiovasc Ther 2010; 28(5):72-91.

6. Gragnano F, Sperlongano S, Golia E, et al. The role of von Willebrand factor in vascular inflammation: from pathogenesis to targeted therapy. Mediators Inflamm 2017; 2017:5620314. Doi: $10.1155 / 2017 / 5620314$.

7. Frankel DS, Meigs JB, Massaro JM, et al. Von Willebrand factor, type 2 diabetes mellitus, and risk of cardiovascular disease: the Framingham Offspring Study. Circulation 2008; 118(24): 25332539.

8. Madan R, Gupt B, Saluja S, et al. Coagulation profile in diabetes and its association with diabetic microvascular complications. J Assoc Physicians India 2010; 58:481-484.

9. Adly AA, Elbarbary NS, Ismail EA, et al. Plasminogen activator inhibitor-1 (PAI-1) in children and adolescents with type 1 diabetes mellitus: relation to diabetic micro-vascular complications and carotid intima media thickness. J Diabetes Complications $2014 ; 28(3): 340-347$.

10. Antoniades C, Bakogiannis C, Tousoulis D, et al. The CD40/CD40 ligand system: linking inflammation with atherothrombosis. J Am Coll Cardiol 2009; 54(8):669-677.

11. Rizvi M, Pathak D, Freedman JE, et al. CD40-CD40 ligand interactions in oxidative stress, inflammation and vascular disease. Trends Mol Med 2008; 14(12):530-538.

12. CarrizoT del R, Prado MM, Velarde MS, et al. Soluble E-selectin in children and adolescents with type 1 diabetes. Medicina 2008; 68(3):193-197.

13. Rabago-Rodríguez R, Gómez-Díaz RA, Tanus-Haj J, et al. Carotid intima-media thickness in pediatric type 1 diabetic patients. Diabetes Care 2007; 30(10):2599-2602.

14. Babar GS, Zidan H, Widlansky ME, et al. Impaired endothelial function in preadolescent children with type 1 diabetes. Diabetes Care $2011 ; 34(3): 681-685$.

15. Romano M, Pomilio M, Vigneri S, et al. Endothelial perturbation in children and adolescents with type 1 diabetes: association with markers of the inflammatory reaction. Diabetes Care 2001; 24(9):1674-1678.

16. Carmassi F, Morale M, Puccetti R, et al. Coagulation and fibrinolytic system impairment in insulin dependent diabetes mellitus. Thromb Res 1992; 67(6):643-654.

17. Blann AD, Lip GY. Endothelial integrity, soluble adhesion molecules and platelet markers in type 1 diabetes mellitus. Diabet Med 1998; 15(8):634-642. 
18. el Khawand C, Jamart J, Donckier J, et al. Hemostasis variables in type I diabetic patients without demonstrable vascular complications. Diabetes Care 1993; 16(8):1137-1145.

19. Sapkota B, Shrestha SK, Poudel S. Association of activated partial thromboplastin time and fibrinogen level in patients with type II diabetes mellitus. BMC Res Notes 2013; 6:485.

20. Targher G, Bertolini L, Zoppini G, et al. Increased plasma markers of inflammation and endothelial dysfunction and their association with microvascular complications in type 1 diabetic patients without clinically manifest macroangiopathy. Diabet Med 2005; 22(8):999-1004.

21. Binay $C$, Bozkurt Turhan A, Simsek E, et al. Evaluation of coagulation profile in children with type 1 diabetes mellitus using rotational thromboelastometry. Indian J Hematol Blood Transfus $2017 ; 33(4): 574-580$.

22. Behl T, Velpandian T, Kotwani A. Role of altered coagulationfibrinolytic system in the pathophysiology of diabetic retinopathy. Vascul Pharmacol 2017; 92:1-5.
23. Lemkes BA, Hermanides J, Devries JH, et al. Hyperglycemia: a prothrombotic factor? JThromb Haemost 2010; 8(8):1663-1669.

24. Milne R, Brownstein S. Advanced glycation end products and diabetic retinopathy. Amino Acids 2013; 44(6): 1397-1407.

25. Geraldes $P$, King GL. Activation of protein kinase $C$ isoforms and its impact on diabetic complications. Circ Res 2010; 106(8):1319-1331.

26. EI-Asrar MA, Adly AA, Ismail EA. Soluble CD40L in children and adolescents with type 1 diabetes: relation to microvascular complications and glycemic control. Pediatr Diabetes 2012; 13(8):616-624.

27. Metwalley KA, Farghaly HS, El-Saied AR. Assessment of serum levels of soluble CD40L in Egyptian children and adolescents with type 1 diabetes mellitus: relationship to microalbuminuria and glycemic control. Indian J Endocrinol Metab 2013; 17(6):1024-1029.

28. Targher G, Zoppini G. Soluble CD40L in young type 1 diabetic individuals without clinical microvascular and macrovascular complications. Diabetes Care 2004; 27(5): 1236-1237. 